\title{
Histoire des techniques chirurgicales dans le traitement de l'obésité
}

\section{History of Surgical Techniques for the Treatment of Obesity}

\author{
J. Dargent \\ (C) Lavoisier SAS 2017
}

Si l'obésité est une pathologie bien identifiée depuis l'antiquité, sa forte prévalence est récente, et liée à de nombreux facteurs de civilisation que l'on ne détaillera pas ici. Le sens commun, partagé par la plupart des médecins d'ailleurs, voudrait que pour la très grande part, le comportement alimentaire en soit le responsable, et ce n'est qu'assez récemment que l'on a mis au jour des mécanismes plus complexes. Dès lors, la restriction alimentaire est toujours apparue comme le traitement logique du surpoids, accompagnée de la prise en charge des maladies associées. Comment et pourquoi en est-on venu à considérer l'obésité comme une maladie qui devrait (ou pourrait) faire l'objet d'une thérapeutique agressive, en dehors des états de santé qui en étaient la conséquence ${ }^{1}$, c'est-à-dire une procédure chirurgicale digestive ? Comment cette chirurgie a-t-elle évolué ? Comment a-t-elle conquis progressivement et non sans mal une authentique crédibilité ? Comment at-elle même gagné des lettres de noblesse et trouvé un répondant scientifique de premier plan ? Comment et pourquoi pourrait-elle à présent disparaître ? C'est ce que nous allons examiner en sept épisodes, arbitrairement centrés autour d'une ou plusieurs personnalités, chacun d'eux retraçant une étape importante mise en perspective avec une réplique à l'onde de choc suscitée par la première, ou bien un simple contre-point, le fil narratif cheminant selon un modèle de questions-réponses.

\author{
J. Dargent $(\bowtie)$ \\ Polyclinique Lyon Nord, 65 rue des Contamines, \\ 69140 Rillieux-la-pape, France \\ e-mail : jerome.dargent@polyclinique-rillieux.fr
}

$\overline{1 . \text { Faire perdre }}$ du poids a toujours eu pour corolaire et même pour principal objectif d'améliorer les pathologies associées à l'obésité. Ainsi en 1966, Edward Mason (nous y reviendrons largement) présente-t-il son premier cas de bypass gastrique comme ayant pour but la réparation différée d'une éventration importante, le temps de la perte de poids tenant le rôle d'une préparation à l'intervention-cible.
À celui qui considérerait un horizon séculaire et ne verrait dans l'émergence, la continuation, et peut-être la disparition de la chirurgie de l'obésité, qu'une note en bas de page d'une histoire majestueuse de la médecine, nous répondrons que les changements de perspective qu'elle a amené débordent de loin le cadre de la pensée médicale. C'est un objet dont le déroulement encore à l'œuvre ne cesse de nous surprendre, tant il est riche d'angles multiples et de remises en cause de certitudes médicales. En fouillant les détails de premières publications, des controverses ou des débats parfois corporatistes, on découvre des présupposés et des influences remarquables. Citons l'historien canadien Jean-Philippe Gendron [1] : « Du point de vue de l'histoire des pratiques médicales, la chirurgie de l'obésité relève d'une transformation majeure des horizons d'application du traitement opératoire et fait apparaître une redistribution fondamentale des conceptions scientifiques du corps, de la maladie et de la thérapeutique. »

Partons donc à la découverte de ces techniques surprenantes!

\section{Référence}

1. Gendron JP (2016) Généalogie de la médecine opératoire de l'obésité : une histoire de l'expérimentation en chirurgie gastro-intestinale, XVII-XX ${ }^{\mathrm{es}}$ siècles. Thèse, Université du Québec (Montréal). 